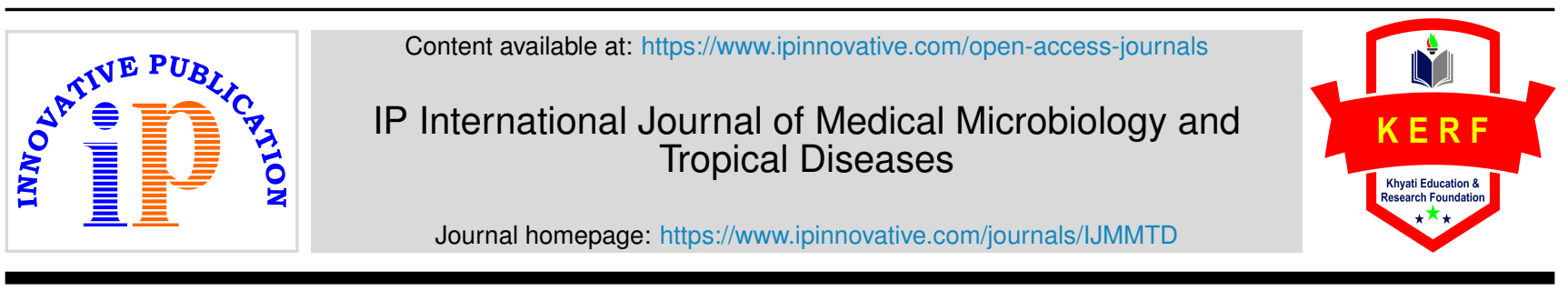

Original Research Article

\title{
Retrospective study of incidence of metallo- $\beta$-lactamase-producing Non-fermenters in nosocomial infections of a tertiary care hospital, in Navi Mumbai
}

\author{
Arjumand Banu' $^{1}$, Rita Swaminathan ${ }^{2}$, Narayana Kamath ${ }^{3} *$, S K Joshi², \\ Ravishkhar K ${ }^{2}$, Abhay Choudhary ${ }^{2}$ \\ ${ }^{1}$ Consultant Microbiologist, Mumbai, Maharashtra, India \\ ${ }^{2}$ Dept of Microbiology, D.Y.Patil Medical College, Navi Mumbai, Maharashtra, India \\ ${ }^{3}$ Dept. of Microbiology, Namo Medical Education \& Research Institute, Dadra and Nagar Haveli, India
}

\section{A R T I C L E I N F O}

\section{Article history:}

Received 11-11-2020

Accepted 01-12-2020

Available online 02-01-2021

Keywords:

Metallo lactamases (MBL)

Nonfermenters

Pseudomonas aeruginosa

Acinetobacter Spp

$\beta$ combined disc test

Nosocomial infection

\begin{abstract}
A B S T R A C T
Background: The growing increase in the rate of antibiotic resistance is a major cause for concern in both non-fermenting bacilli. $\beta$-lactams have been the mainstay of treatment for serious infections, and the most active of these are the carbapenems. Acquired metallo- $\beta$-lactamases (MBL) have recently emerged as one of the most worrisome resistance mechanisms owing to their capacity to hydrolyze all $\beta$-lactams, including carbapenems. We have undertaken this investigation to ascertain the incidence of MBL-producing non-fermenting bacilli, like Pseudomonas Spp. and Acinetobacter Spp.

Aim: To determine the incidence of MBL in Nosocomial infections of non-fermenters and their clinical correlation.

Objectives : Screening of nosocomial isolates for multidrug resistance by antibiotic sensitivity tests as per CLSI guidelines as a reference.

To perform phenotypic confirmatory test of potential MBL isolates by double disc synergy test

Clinical correlation with detection of these enzymes and the patients morbidity/mortality correlation with age, immunocompromised status and hospital stay.

Materials and Methods : The study was conducted over a period of 12 months in a teaching hospital, in Navi Mumbai. Isolates included in the study were screened for imipenem resistance by conventional methods. The isolates that showed imipenem resistance were tested for MBL production by imipenem (IMP)-ethylene-diamine-tetra-acetic acid (EDTA) combined disc test. Imipenem-resistant nonMBL isolates also were tested for Modified Hodge test and AmpC $\beta$-lactamases production to detect other mechanisms of carbapenem resistance.

Results: Total number of isolates tested were 107.Out of 107, 29 (27.1\%) were MBL producers and $78(72.9 \%$ ) were non- MBL producers. Among both types of non-fermenters , 66(61.68\%) were Pseudomonas aeruginosa and 41(32.23\%) were of Acinetobacters. In the sex distribution, 65.5\%were males and $34.5 \%$ were females. $18.18 \%$ were MBL positive Pseudomonas and among Acinetobacter species $41.46 \%$ were MBL positive. Mean age of MBL producers was 34 years. Mean hospital stay was 28 days. Mortality rate was $51.7 \%$ in MBL producers. Mortality was higher in Acinetobacter MBL producers. when compared to Pseudomonas MBL producers.

(C) This is an open access article distributed under the terms of the Creative Commons Attribution License (https://creativecommons.org/licenses/by/4.0/) which permits unrestricted use, distribution, and reproduction in any medium, provided the original author and source are credited.
\end{abstract}

\section{Introduction}

The increase in the rates of antibiotic resistance is a major cause for concern in both non-fermenting bacilli . $\beta$-lactams have been the mainstay of treatment for serious

\footnotetext{
* Corresponding author.

E-mail address: nktallur@gmail.com (N. Kamath).
} 
infections. Most active of these are the carbapenems, which are advocated for use for the treatment of infections caused by extended-spectrum- $\beta$-lactamase (ESBL)producing Enterobacteriaceae, particularly Escherichia coli and Klebsiella pneumonia, and non-fermenters. Among non-fermenters, Pseudomonas spp. and Acinetobacter spp. are commonly isolated.

Five different types of MBLs whose prevalence are increasing rapidly ${ }^{1}$ are IMP(Imipenamse), VIM(Verona Integron-encoded metallo-beta lactamse; Meyrol, SPM1(Sao Paulo Metallo-Brta-lacamase, GIM(German imipenamse; and AIM, Australian Imipenamase; Among these, IMP and VIM (Verona Integron-encoded Metallo Beta lactamase are the most predominant. With the global increase in the occurrence and types of MBLs, early detection is crucial, the benefits of which include timely implementation of strict infection control practices and treatment with alternative antimicrobials. Among the simple and economic methods available for testing MBL production, the imipenem (IMP)-EDTA combined disc test is sensitive and specific. According to (IMP) $10 \mu \mathrm{g}$-EDTA $750 \mu \mathrm{g}$ combined disc test has $95.7 \%$ sensitivity and $91.0 \%$ specificity for detection of metallo-beta-lactamase in MBL-producing Pseudomonas spp and Acinetobacter spp.

Acinetabacter baumanii is a typical nosocomial pathogen causing infections and a high mortality often among patients hospitalized in the Intensive care unit. ${ }^{2}$

Pseudomonas aeruginosa has been described as the most common and seroius cause of infection. ${ }^{3}$

Acquired metallo- $\beta$-lactamases (MBL) have recently emerged as one of the most worrisome resistance mechanisms owing to their capacity to hydrolyze all $\beta$-lactams, including carbapenems. Such strains are not susceptible to therapeutic serine $\beta$-lacatamase inhibitors (such as clavulanate and sulfones). Moreover, their genes are carried on highly mobile elements, allowing easy dissemination. Invasive infections with MBL-producing isolates are also associated with a higher morbidity and mortality. ${ }^{4}$ The occurrence of an MBL-positive isolate in a hospital environment poses not only a therapeutic problem but is also a serious concern for infection control management. Pseudomonas aeruginosa and Acinetobacter baumannii are among the most important causes of healthcare-associated infections. ${ }^{5}$ As a result of being difficult to detect, such organisms pose significant risks, particularly due to their role in unnoticed spread within institutions and their ability to participate in horizontal MBL gene transfer with other pathogens in the hospital. In recent years, MBL genes have spread from $P$. aeruginosa, Acinetobacter Spp. to the members of the Enterobacteriaceae.

Our hospital, a tertiary care centre in Navi Mumbai Maharashtra, has a high prevalence of infections due to nonfermenting bacilli and Enterobacteriaceae. Carbapenems and cephalosporin inhibitor combinations are being used as the "last resort" in these infections since the last few years. This research investigation is undertaken to ascertain the prevalence of MBL-producing non-fermenting bacilli in our hospital. Although a global increase in the prevalence of MBL-producing non-fermenting bacilli has been reported limited data is available from NaviMumbai.

\section{Materials and Methods}

The study was conducted over period of 1 year in our hospital, which is a 1250 bedded teaching hospital with ICUs, trauma centre and one neonatal intensive care unit (NICU). Clinical samples like pus, blood, urine, body fluids, tissues and respiratory secretions from patients admitted in medical, surgical intensive care unit were collected using aseptic precautions. All samples were processed using standard Microbiological procedures.

\subsection{Metallo- $\beta$-lactamase screening}

MBL production was detected in imipenem-resistant isolates by phenotypic tests. The Imipenem (IMP)-EDTA combined disc test was used. ${ }^{5}$

The Imipenem (IMP)-EDTA combined disk test was performed as described by Pseudomonas aeruginosa ATCC 27853 used as the control. Test organisms were inoculated on to plates of Mueller-Hinton agar as recommended by the CLSI. Two 10- $\mu \mathrm{g}$ imipenem disks (Hi-Media Laboratories, BD Diagnostics Pvt. Ltd.) were placed on the plate and appropriate amounts of $10 \mu \mathrm{L}$ of EDTA solution was added to one of them to obtain the desired concentration $(750 \mu \mathrm{g})$. The inhibition zones of the imipenem and Imipenem(IMP)EDTA disks were compared after 16-18 h of incubation in air at $35^{\circ} \mathrm{C}$. In the combined disc test, if the increase in inhibition zone with the imipenem and EDTA disc was $\geq 7 \mathrm{~mm}$ than the imipenem disc alone, it was considered as MBL positive.

\subsection{Antimicrobial susceptibility}

Antimicrobial susceptibility of all the isolates was performed by the Kirby-Bauer disc diffusion method according to the CLSI guidelines. ${ }^{6}$

Antibiotics used are amikacin $(30 \mathrm{mcg})$, gentamicin (10mcg), netilmicin (30mcg), cefoperazone (75mcg), cefipime $(30 \mathrm{mcg})$ ceftazidime $(30 \mathrm{mcg})$ ceftrizoxime (30mcg) ciprofloxacin $(5 \mathrm{mcg})$, lomefloxacin $(10 \mathrm{mcg})$

\section{Results}

\subsection{Bacterial isolates}

A total of 107 gram negative non-fermenting bacteria were isolated from various clinical samples of admitted patients were included in the study.Clinical samples were all clinical branches ans also from patients with co morbid conditions 
like burns, cancer and diabetes.

All isolates were non-duplicate. The isolates were identified by conventional methods. Out of 107 samples, 51 were from pus, 18 from tracheal aspirates, 22 from urine, 8 from sputum and 10 from blood culture.

Pseudomonas aeruginosa was isolated from $66(61.8 \%)$ samples and 41(38.3\%) are from Acinetobacter species.19(65.5\%) males and 10(34.5\%) females were MBL producers, showing a male preponderance. 12 out of 66(18.18\%)were MBL producing Pseudomonas.aeruginosa. and 17 out of $41(41.46 \%)$ were Acinetobacter species. Mean age of MBL producers were 34 years

Mean patients age of MBL producers - 34 years.

\subsection{Antibiotic sensitivity of MBL positive Pseudomonas aeruginosa}

Collostin (100\%), Gatifloxacin (50\%), Piperacillintazobactam (33.3\%),Chloramphenicol (33.3\%) and Netilmicin (16.66\%)

\subsection{Antibiotic senstivity of MBL positive Acinetobacter spp}

Colistin (70.6\%), Gatifloxacin (17.3\%) and piperacillin tazobactam (11.6\%).

All $29 \mathrm{MBL}$ isolates tested positive by the combined disc (Imipenem + EDTA ) method

Out of $29 \mathrm{MBL}$ isolates screened positive by the combined disc method,4(13.7\%) were non determinable by the E-test MBL

Out of the remaining $25(86.2 \%)$ that tested positives by the E-test MBL, 6 showed deformation of the ellipse.

Mean hospital stay was 28 days in the MBL producers.

Infection associated with hospital related devices was 17 out of the 29,(58.6\%) MBL producers. Of these 17,12 (70.5\%) were Acinetobacter spp. Ans 5(29.5\%) were Pseudomonas aeruginosa.

Mortality was seen only in MBL producers, $15(51.7 \%)$ out of 29 succumbed to death.

Mortality is higher in Acinetobacter MBL producers, 12 out of $17,(70.5 \%)$ as compared to Pseudomonas aeruginosa 3 out of $12,(20.5 \%)$.

Highest mortality is seen in infections due to hospital related devices, 6 out of $15(40 \%)$, followed by multi-organ damage 4 out of 15(26.4\%), septicemia 3 out of $15(20 \%)$ and renal failure 2 out of $15(13.3 \%)$.

Table 1: Total number of isolates:

\begin{tabular}{ccc} 
MBL & Non-MBL & Total \\
29 & 78 & 107 \\
27.1 & 72.89 & $100 \%$ \\
\hline
\end{tabular}

\begin{tabular}{lc}
\hline Parameter & Value \\
$\mathrm{F}(2,3)$ & 221.6931 \\
$\mathrm{p}$-value & 0.0006 \\
\hline
\end{tabular}

Table 2: Sex wise distribution

$\begin{array}{ccc}\text { Sex } & \text { MBL } & \text { Non MBL } \\ \text { Male } & 19 & 41 \\ \text { Female } & 10 & 35\end{array}$

Table 3: Patients Mortality rate and bacteria isolated

\begin{tabular}{lccc}
\hline & Pseudomonas & Acinetobacter & $\mathbf{n}$ \\
$\mathrm{n}$ & 66 & 41 & 107 \\
Moratlity & 3 & 12 & 15 \\
Percentage & $2.8 \%$ & $11.21 \%$ & $14.01 \%$ \\
\hline
\end{tabular}

Table 4: Clinical reason causing mortality in MBL producers

\begin{tabular}{cccc}
\hline $\begin{array}{c}\text { Device } \\
\text { related }\end{array}$ & $\begin{array}{c}\text { Multi organ } \\
\text { Damage }\end{array}$ & $\begin{array}{c}\text { Renal } \\
\text { failure }\end{array}$ & Septicemia \\
6 & 4 & 2 & 3 \\
$5.6 \%$ & $3.7 \%$ & $1.86 \%$ & $2.8 \%$ \\
\hline
\end{tabular}

Table 5: Age wise distribution

\begin{tabular}{lccccc}
\hline Age & $\mathbf{1 2 - 5}$ & $\mathbf{2 8 - 4 3}$ & $\mathbf{4 3 - 5 9}$ & $\mathbf{6 0 - 7 4}$ & Above 75 \\
& $\mathbf{2 7}$ & & & & \\
MBL & 2 & 15 & 3 & 5 & 4 \\
Non & 5 & 20 & 2 & 17 & 24 \\
MBL & & & & & \\
\hline
\end{tabular}

Table 6: Other Co-morbid conditions of the MBL isolates

\begin{tabular}{cccc} 
Burns & Cancer & Diabetes & $\begin{array}{c}\text { Infections associated } \\
\text { with Hospital Devices }\end{array}$ \\
2 & 2 & 8 & 17 \\
1.86 & 1.86 & 7.47 & 15.88 \\
\hline
\end{tabular}

\section{Discussion}

MBLs have been found in number of bacteria including Pseudomonas aeruginosa and Acinetobacter species. And there is no CLSI recommended methodology for their detection though simple techniques like combined disc test are available. Molecular typing(genotypic detection) ${ }^{7}$ would help in knowing that which alleles of the genes of MBL's are prevalent in different parts of the world. The diagnostic laboratories worldwide need in house standardized phenotypic tests for their detection Multi-drug resistant or pan drug resistant strains are increasingly being found in hospital settings. MBL producing Pseudomonas aeruinosa are responsible for nosocomial outbreaks across the world. ${ }^{8}$ Treatment options for these organisms are very limited which needs urgent attention by pharma companies to find new molecule.

Acinetobacter baumanii is an important cause of healthcare associated infections and are difficult to treatas 
they possess antimicrobial resistance. ${ }^{9}$ Acquired metallo bete lacatamses ahev recently emerged as one of the most worrisome resistant mechanisms. ${ }^{10}$ Acinetobacter Spp playa significant role in the colonization and infection of patients admitted in the hospitals. ${ }^{11}$

The mainstay of curbing this dreaded menace would be strict infection control practices and restricted use of antibiotics. As in critical care units worldwide,the dictum is hit high,hit hard so start off a patient in ICU with imipenem or Meropenem. But a culture profile with an antibiotic sensitivity must be sent immediately and deescalate the antibiotic according to the report.Cost effective technique is necessary to prevent their dissemination as well as formulation and regulation of antimicrobial stewardship policy. ${ }^{12}$ All Microbiologists, infectious disease specialists and who ever involved in infection control committee of the every hospital should undergo such antimicrobial stewardship program to gain updated knowledge to curb such menaces.A.baumannii has been enlisted as one of the top priority pathogens by World Health Organization in 2017. 13

We have used the combined disc method to be very sensitive for routine detection of MBL's in the laboratory. It is very easy, economical and reliable. The E-test MBL on the other hand is specifically designed to detect as many clinically significant $\mathrm{MBL}$ as possible. The Etest MBL was reliable for detecting the IMP-1- and VIM2-producing Pseudomonas and Acinetobacter isolates. ${ }^{14}$ However given the cost constrains of E-test, the combined disc method is a better option for routine testing in the clinical microbiology laboratories which are Incorporated in many of the laboratories.

Hospital infection control committee and antibiotic policy ${ }^{15}$ makers at hospital level, District, state, national ${ }^{16}$ and international level has to come together and device a concrete policy to treat patients as per the policy to minimize the mortality.In India we should have our own country specific policy and everyone has to follow this without any compliance.

\section{Conclusion}

Our findings show that there are significant numbers of isolates showing MBL production along with MDR. There is a need for active surveillance to detect MBL producers. The Imipenem(IMP)-EDTA combined disc test is a simple test that can be used in district health laboratories or where molecular diagnostic techniques are not available to detect these types of resistance. There should be judicious use of carbapenems to prevent their spread and use of effective antibiotics as per the antibiotic-sensitivity report.

\section{Source of Funding}

No financial support was received for the work within this manuscript.

\section{Conflict of Interest}

The authors declare they have no conflict of interest.

\section{References}

1. Halat DH, Moubareck CA. Antibiotic resistance Science Direct,Comprehensive Natural Products II. 2016;

2. Kabbaj H, Seffar M. Prevalence of Metallo B-lacatmaes producing Acoinetaobacter baumanni in a Moroccan Hospital. Int Scholarly Res Notices. 2013;p. 3.

3. Banjare B, Barapatre R. Incidence of carbapenem-resistant pseudomonas aeruginosa in clinical samples. Int $\mathrm{J}$ Biomed Res. 2015;6(8):567. do1:10.7439/1]br.v618.2418.

4. Yogeesha KV, Jayasimha VL, Basavarajappa KG, Arun K, Raghu KK, Niranjan HP, et al. A comparative study of ventilator associated pneumonia and ventilator associated tracheobronchitis: Incidence, outcome and risk factors. Biosci Biotech Res Asia. 2011;8:195-203.

5. Guzel M, Afsar Y, Akdogan D. Penka Moncheva, Petya Hristova \& Gul Erdem;Evaluation of metallo-beta-lactamase production in multiple antibiotic-resistant Pseudomonas spp. and Acinetobacter baumannii strains. Biotechnol Biotechnological Equipment 2018;32(5):1285-90.

6. CLSI Guidelines

7. Cuong Q, Hoang HD, Nguyen. Emergence of NDM\& KPC in Southern Vietnam,A Cross sectional study. Biomed Res Int.

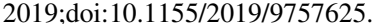

8. Bashir D, Thokar MA. Detection of MBLs producing P.aeriginosa at a tertiary care hospital in Kashmir. Afri J Microbiol Res. 2011;5(2):164 72.

9. Singla P. Co-production of ESBL and AmpC $\beta$-Lactamases in Clinical Isolates of A. baumannii and A. lwoffii in a Tertiary Care Hospital From Northern India. J Clin Diagn Res. 2014;8(4). do1:10.1860//Cdr/2014/8008.4289

10. Deshmukh DG, Damle SA. MBLase producing Clinical isolates from Patients of a Teratiary care Hospital. J Lab Physicains. 2011;3(2).

11. Kaur A, Gupta V. Prevalence of MBL producing Acinetobacter Spp in a tertiary care hospital. Iranain J Microbiol. 2014;6(1).

12. Thapa P, Bhandari D. A Hsopital based survellance of metallo-betalactamse producing gram negative bacteria in Nepal by imipenemEDTA dosk method. BMC Res Notes. 2017;322.

13. World health Organization,WHO publishes list of bacteria for which New Antibiotics are urgentlyneeded,World Health Organization,Geneva ; 2017.

14. Lee K, Yong D. Evaluation of E-test MBL for Detection of blaIMP-1 and blaVIM-2 Allele-Positive Clinical Isolates of Pseudomonas spp. and Acinetobacter spp. J Clin Microbiol. 2005;43(2):942-4.

15. Lakshmi V. Need for national/regional guidelines and policies in India to combat antibiotic resistance. IJMM. 2008;26(2):105-7.

16. Walia K, Ohri VC, Madhumathi J, Ramasubramanian V. Policy document on antimicrobial stewardship practices in India. Indian $J$ Med Res. 2019;149(4):180-4. doi:10.4103/1jmr.ijmr_147_18.

\section{Author biography}

Arjumand Banu, Consultant Microbiologist

Rita Swaminathan, Professor

Narayana Kamath, Professor

S K Joshi, Associate Professor

Ravishkhar K, Professor 
Abhay Choudhary, Professor and HOD

Cite this article: Banu A, Swaminathan R, Kamath N, Joshi SK,

Ravishkhar K, Choudhary A. Retrospective study of incidence of metallo- $\beta$-lactamase-producing Non-fermenters in nosocomial infections of a tertiary care hospital, in Navi Mumbai. IP Int J Med Microbiol Trop Dis 2020;6(4):217-221. 